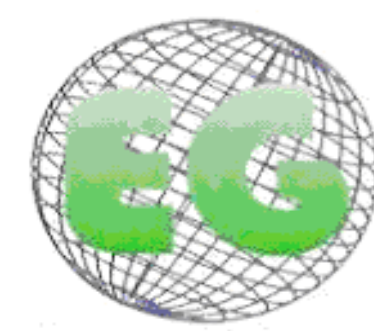

ISSN 1695-6141

$\mathrm{N}^{\circ} 23$
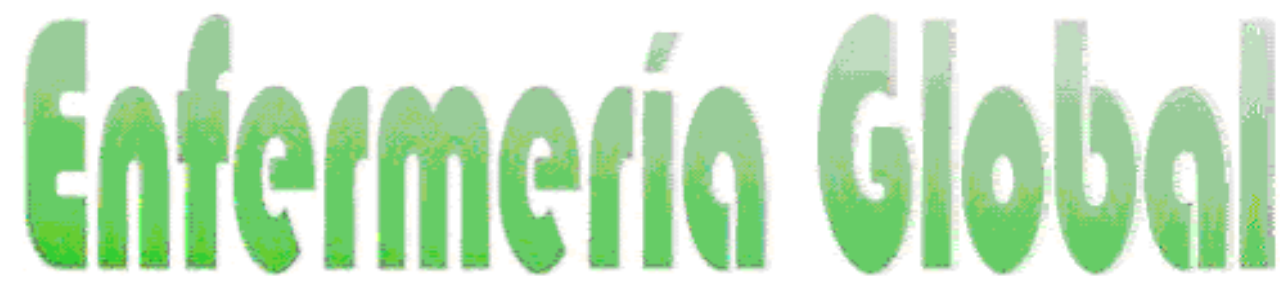

Revista electrónica trimestral de Enfermería

www.um.es/egloball

\title{
Diagnósticos de enfermería en pacientes dados de alta, con enfermedades crónicas, según taxonomía Nanda-I (North American Nursing Diagnosis Association)
}

Nursing diagnoses in patients with chronic diseases, at hospital discharge, by taxonomy Nanda-I.

\author{
*Villarejo Aguilar, Luis \\ *Diplomado en Enfermería. Máster en Investigación e Innovación en Cuidados y Calidad de Vida. Distrito \\ Sanitario Jaén Norte. Atención Primaria. Servicio Andaluz de Salud.
}

Palabras clave: Diagnósticos enfermeros; Enfermedades crónicas; Alta hospitalaria.

Keywords: Nursing Diagnoses; Chronic diseases; Discharge.

\section{RESUMEN}

El presente estudio tiene como objetivo identificar los diagnósticos enfermeros más frecuentes registrados en los Informes de Alta de pacientes con enfermedades crónicas. Se realizó un estudio descriptivo transversal sobre todos los pacientes dados de alta durante el período retrospectivo 20092010. La recogida de datos y análisis de las variables se hizo mediante revisión de los Informes de Alta. Como resultados principales se identificaron que los diagnósticos más frecuentes fueron deterioro de la movilidad física, ansiedad, riesgo de caídas, riesgo de deterioro de la integridad cutánea, intolerancia a la actividad, riesgo de estreñimiento, conocimientos deficientes, dolor agudo y cansancio en el desempeño del rol del cuidador. Existe una importante necesidad de cuidados en los pacientes con enfermedades crónicas derivada de la existencia de problemas activos que precisan continuidad entre niveles asistenciales.

\section{ABSTRACT}

The study aims to identify the most frequent nursing diagnoses recorded on discharge reports for patients with chronic diseases. We conducted a cross sectional study on all patients discharged during the retrospective period 2009-2010. Data collection and analysis of variables was done by reviewing discharge reports. The main results identified that the most common diagnoses were impaired physical mobility, anxiety, risk of falls, risk of impaired skin integrity, activity intolerance, risk of constipation, poor knowledge, acute pain and fatigue in the performance the role of caregiver. There is a significant need for care in patients with chronic diseases resulting from the existence of assets that need continuity problems between levels of care. 


\section{INTRODUCCIÓN}

La acumulación de patologías crónicas en un mismo paciente es cada vez más frecuente, por lo que se está convirtiendo en un problema de salud pública ${ }^{(1)}$. El envejecimiento de la población española, la relación de la comorbilidad con la edad y de ambas con la mortalidad hacen prever que las dimensiones de este problema irán en aumento ${ }^{(2)}$. El aumento de las enfermedades crónicas es una carga creciente y plantea exigencias nuevas y diferentes a los sistemas de salud de todo el mundo. Las enfermedades cardiovasculares, el ictus, las enfermedades respiratorias crónicas, la diabetes de tipo 2, y más de una tercera parte de los cánceres representan una de las principales amenazas para la salud y el desarrollo humano. La magnitud del problema es tal que según estimaciones de la OMS, estas enfermedades son las responsables de 35 millones de fallecimientos al año ${ }^{(3)}$. En multitud de ocasiones, tras largos años de cuidados crónicos permanentes. Lo más dramático es que hasta el $80 \%$ de los casos podrían evitarse eliminando factores de riesgo comunes, principalmente, el uso del tabaco, las dietas no saludables, la inactividad física, o el uso del alcohol.

Ante este panorama, es urgentemente necesario que en todo el mundo las enfermeras tomen la iniciativa para abordar esta amenaza creciente para la salud y el bienestar mundiales. El potencial de la enfermería mediante la atención a la prevención y cuidados de las enfermedades crónicas parte de una gestión y unos cuidados eficaces, desde las primeras fases, para permitir que los afectados por estas enfermedades crónicas lleven una vida plena y productiva ${ }^{(4)}$. El conocimiento de los diagnósticos enfermeros prevalentes es la primera fase en el proceso de estandarización de cuidados. La estandarización nos permitirá unificar criterios de actuación, sistematizar etapas y proporcionar un cuidado individualizado para cada paciente, con un criterio de actuación conjunto ${ }^{(5)}$. Asimismo la aplicación del Proceso de Atención de Enfermería con taxonomías permite la sistematización de las etapas de los cuidados y sirve de guía para evitar la variabilidad de cada profesional ${ }^{(6)}$.

El estudio se plantea con el objetivo de identificar los diagnósticos más frecuentes en pacientes con enfermedades crónicas al alta hospitalaria.

\section{MATERIAL Y MÉTODOS}

- DISEÑO: Descriptivo transversal.

- ÁMBITO Y SUJETOS DE ESTUDIO: El ámbito de estudio será un Hospital de tercer nivel y los sujetos de estudio serán todos los pacientes ingresados en el Hospital a los que se les haya hecho el Informe de Alta durante el período 2009-2010.

- RECOGIDA DE DATOS: Todos los datos serán recogidos de los Informes de Alta.

- ANÁLISIS DE DATOS: Análisis descriptivo univariante con frecuencias y porcentajes para variables categóricas y medias y desviación estándar para variables cuantitativas.

- LIMITACIONES: La principal limitación del estudio es el sesgo de selección a la hora de seleccionar los pacientes con enfermedades crónicas a partir de la causa principal que originó su ingreso hospitalario y del proceso asistencial integrado. También el hecho de que la muestra sólo se ha tomado de un Hospital de referencia, pero de distintas Zonas Básicas lo que afectará a la validez externa del estudio. 


\section{RESULTADOS}

Como resultado del análisis de los diagnósticos presentes en los planes de cuidados de los Informes de Alta de la muestra estudiada se han identificado 30 diagnósticos distintos, se han formulado un total de 581 diagnósticos, lo que supone una media de 3,85 diagnósticos por paciente $\pm 1,79$ ( desviación estándar). El número de diagnósticos al alta osciló, en cada caso, entre 1 y 9 diagnósticos. Gráfico 1-

Gráfico 1. Número de Diagnósticos registrados en los Informes de Alta de pacientes con enfermedades crónicas.

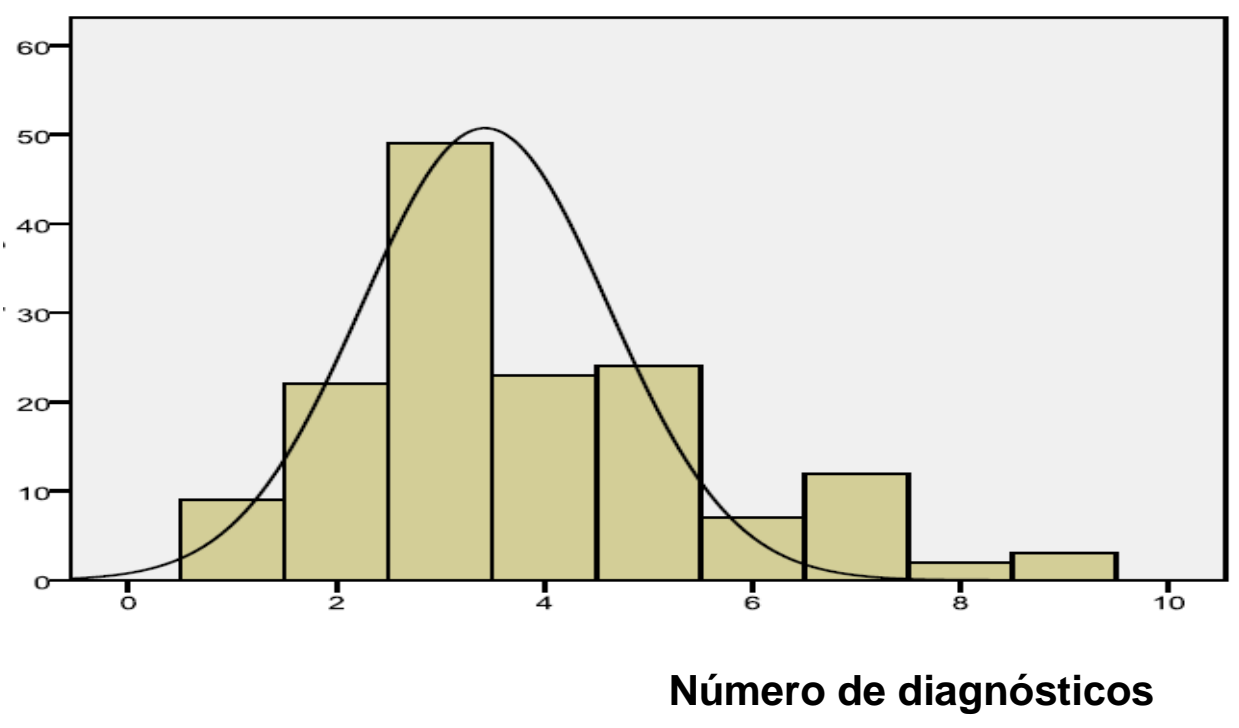

Los diagnósticos más frecuentes (repetidos 10 o más veces) fueron los siguientes: deterioro de la movilidad física $50,3 \%$, ansiedad $50,3 \%$, riesgo de caídas $43,0 \%$, riesgo de deterioro de la integridad cutánea $36,4 \%$, intolerancia a la actividad $34,4 \%$, riesgo de estreñimiento $31,8 \%$, conocimientos deficientes $21,2 \%$, dolor agudo $18,5 \%$, cansancio en el desempeño del rol del cuidador $15,2 \%$, desequilibrio nutricional por defecto $13,2 \%$, manejo inefectivo del régimen terapéutico $12,6 \%$, riesgo de cansancio en el desempeño del rol de cuidador $11,9 \%$, afrontamiento familiar comprometido 11,3\%,y déficit de autocuidados: alimentación 6,6\%. Gráfico 2. 
Gráfico 2. Diagnósticos de Enfermería presentes en los Informes de Alta de pacientes con enfermedades crónicas.

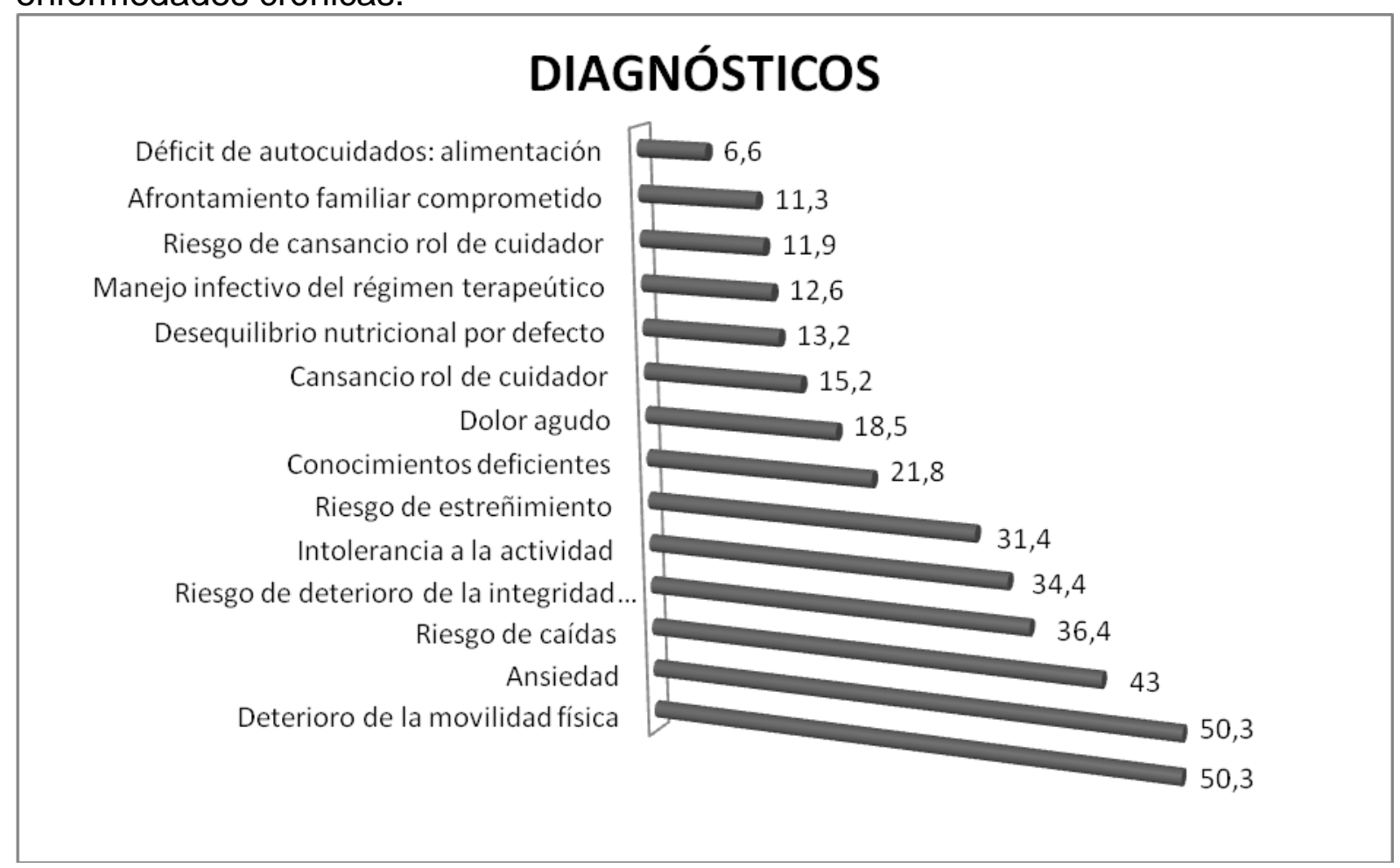

\section{DISCUSIÓN}

En el presente estudio se confirma que en el momento del alta hospitalaria, el colectivo de pacientes con enfermedades crónicas presenta una importante demanda de cuidados derivada de la existencia de problemas activos que precisan seguimiento por las enfermeras de atención primaria, para garantizar la continuidad de cuidados.

Pese a la variabilidad de diagnósticos encontrados podemos decir que existen unos diagnósticos más frecuentes, de tal manera que los 10 primeros diagnósticos, ordenados según su frecuencia, suponen prácticamente el $81 \%$ del total de diagnósticos. Entre ellos están el deterioro de la movilidad física, ansiedad, riesgo de caídas, riesgo de deterioro de la integridad cutánea, intolerancia a la actividad, riesgo de estreñimiento, conocimientos deficientes, dolor agudo, cansancio en el desempeño del rol del cuidador y desequilibrio nutricional por defecto.

En nuestra opinión la mayor frecuencia de diagnósticos enfermeros utilizados pertenecen a problemas fisiológicos y cognitivo-conductuales; se deben en parte al alto grado de fragilidad que presentan estos pacientes y por otra parte a la priorización por parte de los profesionales de los problemas biofísicos. Estos datos coinciden con otros estudios ${ }^{(7,8,9)}$ que afirman el elevado número de enfermeras que utilizan los diagnósticos que le son más propios y que habitualmente trabajan.

El número medio de diagnósticos al alta 3,85 encontrado en nuestro estudio coincide con el encontrado por Zambrana García JL, et al. quienes hallaron 3,6 de media y el $42 \%$ de los pacientes contaban con más de tres diagnósticos al alta ${ }^{(10)}$ y difieren de los encontrados por Gallego et al. que hallaron una media de 6 diagnósticos por paciente ${ }^{(11)}$ y Clavería que también identificó una media de 6,3 diagnósticos por paciente ${ }^{(7)}$. 


\section{CONCLUSIONES}

Como conclusión señalar que el presente estudio contribuye a subrayar la importancia de una adecuada valoración enfermera al alta, de cara a establecer estrategias preventivas y de seguimiento de estos pacientes con enfermedades crónicas, para promover los autocuidados y una gestión de los cuidados de manera dinámica, integrada y planificada para evitar el deterioro y reingresos innecesarios.

\section{BIBLIOGRAFÍA}

(1) Van den Akker M, Buntinx F, Roos S, et al. Problems in determining occurrence rates of multimorbidity. J Clin Epidemiol. 2001; 54(7): 675-9.

(2) Cantalapiedra M. El envejecimiento demográfico en España. Fuentes Estadísticas. 2002; 68.

(3) Organización Mundial de la Salud.OMS.

(4) Consejo Internacional de Enfermeras.CIE. Con motivo del Día Internacional de la Enfermería. Madrid, 12 de mayo 2010.

(5) Alfaro,R. Aplicación del proceso de enfermería (2 $2^{\underline{a}}$ ed.). Barcelona: Doyma Enfermería, 1992.

(6) Fuchs E, Marín I, Ponsich F, Martinez P. Estandarización de cuidados en una planta de rehabilitación. Enfermería Global. 1995; (4).disponible en http://www.um.es/eglobal/4/04d01.htlm.

(7) Clavería M. Diagnósticos de enfermería utilizados por un equipo de atención domiciliaria socio-sanitaria. Enfermería Científica. 1997; 182-83:49-54.

(8) Álvarez I, Ohara R, Casado E, Castañeda E, Galán JM, Morilla JC. Diagnósticos aplicados a la población incapacitada. Rev Rol Enferm 1997; 226:25-8.

(9) Brito Brito, PR. Diagnósticos enfermeros priorizados en atención primaria. Rev. Enfermería Global 2009; 16: 1-8.

(10) Zambrana García JL, Velasco Malagón MJ, Díez García F, Cruz Caparrós G, Martín Escalante MD, Adarraga Cansino, MD. Características clínicas diferenciales de los enfermos pluripatológicos hospitalizados en servicios de Medicina Interna. Rev Clin Esp. 2005; 205(9):413-7.

(11) Gallego P, Carra B, Morente M, Calvo C, Sanz C. Visita domiciliaria. Diagnósticos médicos y de enfermería. Rev Rol Enferm 1995; 202:14-8.

ISSN 1695-6141

@ COPYRIGHT Servicio de Publicaciones - Universidad de Murcia 